\title{
COMPETITIVIDAD DE LAS EXPORTACIONES DE ACEITUNA DE MESA (OLEA EUROPAEA L.) CHILENA EN EL MERCADO BRASILEÑO ENTRE LOS AÑOS 1993-2005
}

\author{
COMPETITIVENESS IN EXPORTS OF THE CHILEAN OLIVE \\ IN THE BRAZILIAN MARKET BETWEEN 1993 AND 2005
}

\author{
Esteban Vargas Masquimillan ${ }^{1}$; Luis Tapia Iturrieta ${ }^{1}$
}

\section{RESUMEN}

\begin{abstract}
Chile encabeza la lista de países más competitivos de América Latina y el Caribe, según los resultados del Índice de Competitividad Latinoamericano 2001-2002 publicado por el Foro Económico Mundial.

Esto se ve evidenciado en el sostenido crecimiento del sector agrícola, que en las últimas dos décadas tiene posicionado a Chile entre los mayores exportadores de fruta del hemisferio sur, situación que no se ve reflejada en el sector olivícola. Al considerar las exportaciones de aceituna de mesa al mercado brasileño, se observa una reducción sistemática que preocupa por ser éste el principal mercado para de las exportaciones de aceituna de mesa chilena y además se registra un retroceso en comparación a la participación de los competidores que también exportan a este importante mercado.
\end{abstract}

Palabras clave: Principales países exportadores olivícolas al mercado brasileño, exportaciones olivícolas chilenas, competitividad.

\begin{abstract}
Chile is heading the list of the most competitive countries in Latin America and the Caribbean, according to the results in the Latin America Competitiveness Index 2001-2002 published by the Wold Economy Forum.

This is evidenced in the sustained growth of the agriculture, which during the last two decades has Chile among the biggest fruit exporters in the south hemisphere, but it is not evidenced in the olive market. Considering the olive exports to the Brazilian market it is observed that there is a systematic reduction, which is a concern because Brazil is the main market for the Chilean olive. Besides, it shows a setback in comparison with the competence.
\end{abstract}

Key words: main olive export countries to the Brazilian market, chilean olive exports, competitiveness.

\section{INTRODUCCIÓN}

Un país es competitivo cuando puede ocupar sus recursos eficientemente para incrementar los ingresos y aumentar el nivel de desarrollo de sus habitantes. La competitividad en el sector agrícola se ve demostrada por el desarrollo de nuevas tecnologías que permitan una mayor producción y una disminución en los costos de los procesos productivos.

En el caso de las exportaciones de aceitunas chilenas de mesa, en la última década se ha man- tenido una tendencia negativa a las importaciones de este producto por parte de Brasil, considerado el mayor importador de la región FAO (1993-2005). Esto se podría deber a distintos factores, como el aumento de las importaciones desde otros países productores al estancamiento tecnológico que vive el sector olivícola chileno, que impacta sobre la calidad del producto, el precio de la aceituna chilena con relación al precio ofrecido por los otros países competidores directos que acceden a este mercado, entre otros.

1 Departamento de Producción Agrícola, Facultad de Ciencias Agronómicas de la Universidad de Tarapacá, Arica, Chile, E-mail: 1tapia@uta.cl 
Estos factores están afectando las exportaciones de aceituna de mesa a Brasil. Por otro lado, la importancia que tiene el mercado brasileño para la aceituna chilena es enorme, ya que Brasil representa el 63\% de las exportaciones de aceituna de mesa. Seguido por las exportaciones a EE.UU. con un $11,6 \%$, América del Sur reúne el $72 \%$ del destino de las exportaciones de aceituna de mesa chilena.

Dadas las favorables condiciones que tiene Chile y especialmente la zona norte para la producción de aceitunas es importante conocer las razones que explican la disminución de las exportaciones, ya que la aceituna sigue siendo un producto valorado en el resto del mundo, con una importante expansión del sector de las aceitunas de mesa. A diferencia de la producción de aceite de oliva, la aceituna de mesa es mucho más estable C.O.I. (2005).

Al hablar de la disminución de las exportaciones de aceituna de mesa también se puede mencionar la disminución de la producción debido al añerismo, el cual es atribuido a las variaciones en la cantidad de frío invernal, como principal factor climático Tapia (1985).

Según Guerrero (1997), la floración y fructificación guardan relación con el número de horas frío que pasa el olivo. El número de horas frío requerido para una floración máxima cambia con la variedad.

\section{MATERIALES Y MÉTODOS}

El tipo de estudio que se ha planteado para estimar la competitividad del sector olivícola exportador de aceituna de mesa al mercado brasileño es de tipo descriptivo, porque tiene como objetivo indagar la incidencia y los valores que se manifiestan con cada una de las posibles variables. Es una investigación no experimental, ya que se realiza sin alterar intencionalmente las variables estudiando éstas tal como se dieron en su contexto natural, Hernández et al. (2003).

El propósito del estudio descriptivo es medir, evaluar o recolectar información sobre diversos aspectos, dimensiones o componentes del fenómeno a investigar (Hernández et al., 2003).

El diseño de la investigación es de tipo documental, con medición de variables a partir de datos secundarios. El propósito es conocer las variables que afectan la competitividad de la aceituna de mesa chilena que se exporta al mercado brasileño.
El estudio de la competitividad está basado en el análisis de series de tiempo donde se evalúan las tendencias, estacionalidad y precios entre los años 1993-2005.

Además se presenta un análisis de regresión múltiple para evaluar la correlación entre las variables. También se evalúa la relación entre las variables independientes, utilizando los coeficientes múltiples de determinación (Little y Hills, 1976).

El estudio y análisis de la información recopilada se utilizó para establecer las series de tiempo entre los años 1993-2005, tanto para las importaciones por parte del mercado brasileño como para las exportaciones de Chile a diferentes mercados.

Esta información se recopiló a partir de las bases de datos de ODEPA y FAO 1993-2005. La información se presenta en forma de tablas y gráficos a fin de determinar la tendencia que muestran tanto las importaciones del mercado brasileño como el comportamiento de Chile y los otros países competidores que acceden a este mercado.

\section{La hipótesis de trabajo es la siguiente:}

¿Está preparado el sector olivícola chileno (productor de aceituna de mesa) para competir exitosamente con los distintos exportadores de aceituna de mesa al mercado brasileño?

\section{RESULTADOS}

Los distintos antecedentes que permiten explicar la competencia que enfrenta la aceituna de mesa chilena al mercado brasileño se presenta a continuación:

- Principales países exportadores al mercado brasileño.

- Análisis de la tendencia de las exportaciones de aceituna, de mesa al mercado brasileño.

- Participación de las exportaciones de aceituna de mesa chilenas al mercado brasileño y su relación con las exportaciones totales de aceituna de mesa de este país.

- Comportamiento de las exportaciones de aceituna, de mesa argentinas y chilenas.

- Comportamiento de las exportaciones de aceituna, de mesa peruanas y chilenas.

- Comportamiento de las exportaciones de aceituna, de mesa española, portuguesa y chilena.

- Tendencias de los precios. 
La Tabla 1 muestra a los principales exportadores de aceituna de mesa al mercado brasileño, donde se puede observar la gran diferencia que existe entre Argentina y el resto de los exportadores mundiales de aceituna que acceden al mercado brasileño. Entre éstos se encuentra Chile, que no destaca por colocar grandes volúmenes de aceituna de mesa.

Las exportaciones de Argentina promedian entre los años 1993-2005, 39.792 t, siendo Perú el país que le sigue en ese mismo periodo con una media de $3.287 \mathrm{t}$, lo cual muestra el gran avance que han tenido las exportaciones peruanas superando a importantes países productores olivícolas como España y Portugal, en la conquista del cada vez más importante mercado brasileño.

La Tabla 2 muestra en forma porcentual las importaciones de aceituna de mesa al mercado brasileño. En ésta se puede observar la gran diferencia que existe entre las exportaciones argentinas de aceituna de mesa al mercado brasileño, a diferencia del resto de los países exportadores, llegando este país a tener un $92 \%$ de las exportaciones de aceituna de mesa al mercado brasileño en el año 1993. En contraste con Chile que su máximo fue sólo de un $3,49 \%$ de las exportaciones de aceituna de mesa al mercado brasileño en el año 1996.

En la Figura 1 se representan las exportaciones de aceituna de mesa chilena al mercado brasileño entre los años 1993-2005. La tendencia negativa muestra la baja que han tenido las exportaciones de aceituna. Además en el gráfico se puede observar lo inestable que han sido los valores exportados de aceituna de mesa en el periodo de tiempo ya señalado.

El coeficiente de correlación de 0,33 indica una correlación baja entre la variable volúmenes de aceitunas de mesa chilena exportada al mercado brasileño y el tiempo en que se realizan estas exportaciones. Este valor no es significativo a ningún valor de confianza, Little y Hills (1976).

En la Figura 2 se pueden observar las exportaciones totales de aceituna chilena y las importaciones brasileñas de aceituna de mesa desde Chile. De esta forma se puede ver gráficamente la importancia del mercado brasileño, siendo éste el principal importador de aceitunas chilenas. Además se observa que las importaciones a través de los años han sido homo-

TABLA 1

Principales países exportadores de aceituna de mesa al mercado brasileño entre los años 1993-2005 (Expresado en toneladas).

\begin{tabular}{|c|c|c|c|c|c|c|c|c|}
\hline Años & $\begin{array}{c}\text { Argentina } \\
(\mathbf{t})\end{array}$ & $\begin{array}{c}\text { Chile } \\
(\mathbf{t})\end{array}$ & $\begin{array}{c}\text { España } \\
(\mathbf{t})\end{array}$ & $\begin{array}{c}\text { Grecia } \\
(\mathbf{t})\end{array}$ & $\begin{array}{c}\text { Italia } \\
(\mathbf{t})\end{array}$ & $\begin{array}{c}\text { Líbano } \\
(\mathbf{t})\end{array}$ & $\begin{array}{c}\text { Perú } \\
(\mathbf{t})\end{array}$ & $\begin{array}{c}\text { Portugal } \\
(\mathbf{t})\end{array}$ \\
\hline 1993 & 34.581 & 1.006 & 308,8 & 243,7 & 3,1 & 31,5 & 443,8 & 837 \\
\hline 1994 & 58.468 & 1.609 & 1.186 & 484,6 & 21,2 & 0 & 599 & 1.447 \\
\hline 1995 & 35.199 & 1.413 & 3.542 & 844,2 & 40,7 & 6,4 & 1.105 & 1.403 \\
\hline 1996 & 36.675 & 1.531 & 2.821 & 154,2 & 32,9 & 16,5 & 2.577 & 2.593 \\
\hline 1997 & 37.233 & 564,1 & 3.321 & 29,4 & 39,4 & 8,2 & 3.534 & 1.790 \\
\hline 1998 & 40.986 & 1.600 & 3.244 & 39,9 & 13,5 & 2,9 & 2.878 & 2.508 \\
\hline 1999 & 40.296 & 354,2 & 1.460 & 96,3 & 12,5 & 11,3 & 1.044 & 1.314 \\
\hline 2000 & 32.980 & 846,8 & 8.192 & 106,25 & 98,39 & 3,7 & 4.343 & 1.618 \\
\hline 2001 & 29.940 & 745 & 5.256 & 103 & 13 & 8 & 5.918 & 1.579 \\
\hline 2002 & 36.909 & 867 & 4.530 & 22 & 6 & 3 & 4.589 & 1.065 \\
\hline 2003 & 40.727 & 1.621 & 322 & 35 & 6 & 0 & 5.012 & 401 \\
\hline 2004 & 46.687 & 1.214 & 835 & 0 & 1 & 7 & 4.284 & 612 \\
\hline 2005 & 47.306 & 424 & 1.297 & 11 & 1 & 3 & 6,406 & 594 \\
\hline Media & 39.792 & 974 & 2.793 & 166 & 22,2 & 7,8 & 3.287 & 1.366 \\
\hline
\end{tabular}

Fuente: Elaboración propia sobre la base de cifras de ODEPA y FAO 1993-2005. 
Tabla 2

Principales países exportadores de aceituna de mesa al mercado brasileño entre los años 1993-2005 (Expresado en porcentaje)

\begin{tabular}{|c|c|c|c|c|c|c|c|c|}
\hline Años & $\begin{array}{c}\text { Argentina } \\
(\boldsymbol{\%})\end{array}$ & $\begin{array}{c}\text { Chile } \\
(\boldsymbol{\%})\end{array}$ & $\begin{array}{c}\text { España } \\
(\boldsymbol{\%})\end{array}$ & $\begin{array}{c}\text { Grecia } \\
(\boldsymbol{\%})\end{array}$ & $\begin{array}{c}\text { Italia } \\
(\boldsymbol{\%})\end{array}$ & $\begin{array}{c}\text { Líbano } \\
(\boldsymbol{\%})\end{array}$ & $\begin{array}{c}\text { Perú } \\
(\boldsymbol{\%})\end{array}$ & $\begin{array}{c}\text { Portugal } \\
(\boldsymbol{\%})\end{array}$ \\
\hline 1993 & 92,32 & 2,68 & 0,82 & 0,65 & 0 & 0,08 & 1,18 & 2,23 \\
\hline 1994 & 91,61 & 2,52 & 1,85 & 0,75 & 0,03 & 0 & 0,93 & 2,26 \\
\hline 1995 & 80,81 & 3,24 & 8,13 & 1,93 & 0,09 & 0,01 & 2,53 & 3,22 \\
\hline 1996 & 83,71 & 3,49 & 6,44 & 0,35 & 0,07 & 0,03 & 5,88 & 5,58 \\
\hline 1997 & 80,03 & 1,21 & 7,14 & 0,06 & 0,08 & 0,017 & 7,59 & 3,84 \\
\hline 1998 & 83,81 & 3,32 & 6,74 & 0,08 & 0,02 & 0 & 5,98 & 5,05 \\
\hline 1999 & 90,37 & 0,79 & 3,27 & 0,21 & 0,02 & 0,02 & 2,34 & 2,94 \\
\hline 2000 & 68,43 & 1,75 & 16,99 & 0,22 & 0,20 & 0 & 9,01 & 3,35 \\
\hline 2001 & 68,72 & 1,71 & 2,06 & 0,23 & 0,029 & 0,01 & 13,58 & 3,62 \\
\hline 2002 & 76,90 & 1,80 & 9,43 & 0,04 & 0,012 & 0 & 9,56 & 2,21 \\
\hline 2003 & 84,62 & 3,36 & 0,66 & 0,07 & 0,012 & 0 & 10,41 & 0,83 \\
\hline 2004 & 87,03 & 2,26 & 1,55 & 0 & 0 & 0,01 & 7,98 & 1,14 \\
\hline 2005 & 85,99 & 0,75 & 2,35 & 0,01 & 0 & 0 & 11,64 & 1,05 \\
\hline PROM & 82,79 & 2,22 & 5,1869 & 0,35 & 0,04 & 0,01 & 6,81 & 2,83 \\
\hline
\end{tabular}

Fuente: Elaboración propia sobre la base de cifras de ODEPA y FAO 1993-2005.

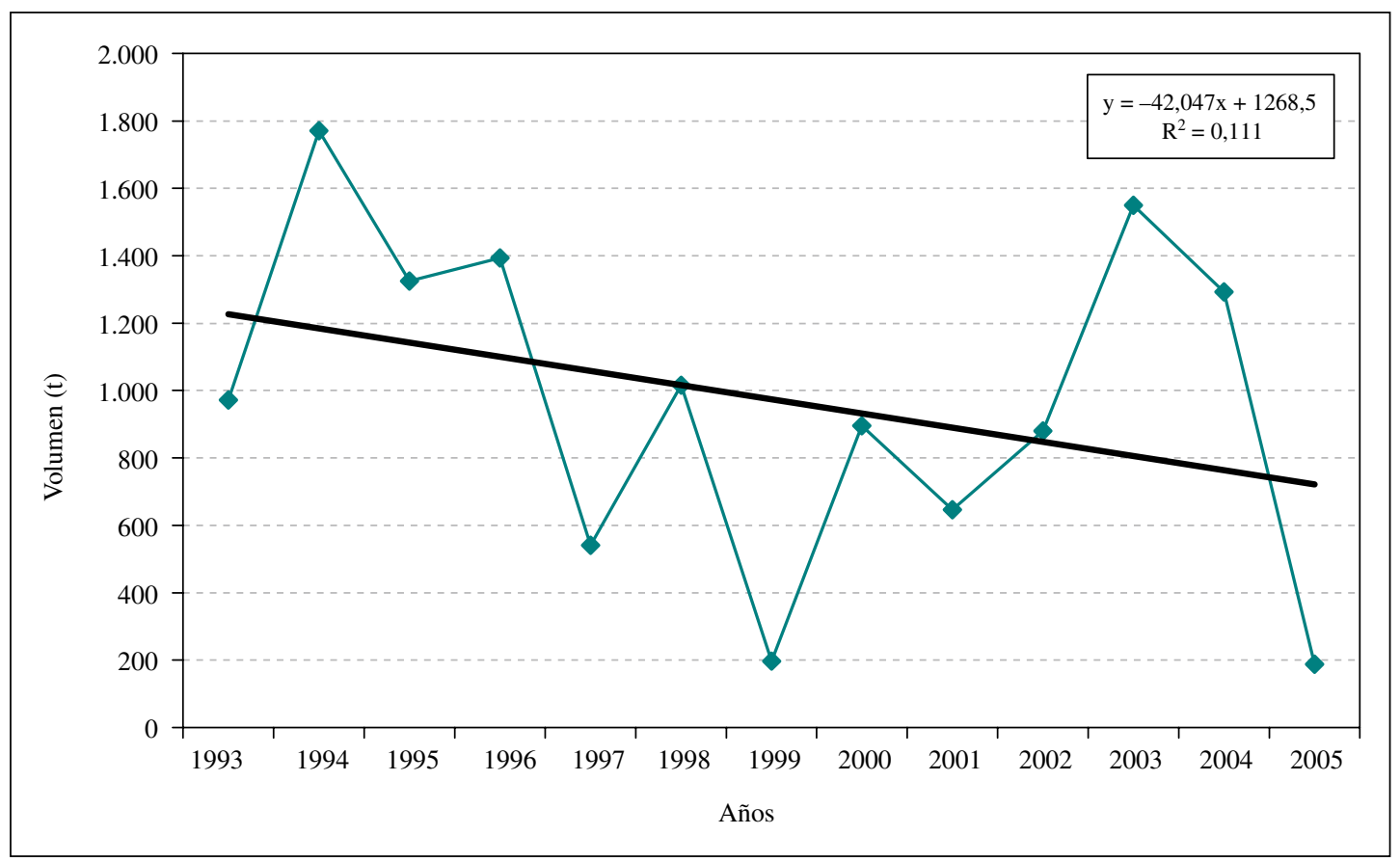

Figura 1. Exportaciones chilenas de aceituna de mesa al mercado brasileño entre los años 1993-2005 (expresado en toneladas). Fuente: Elaboración propia sobre la base de cifras de ODEPA. 1993-2005. 


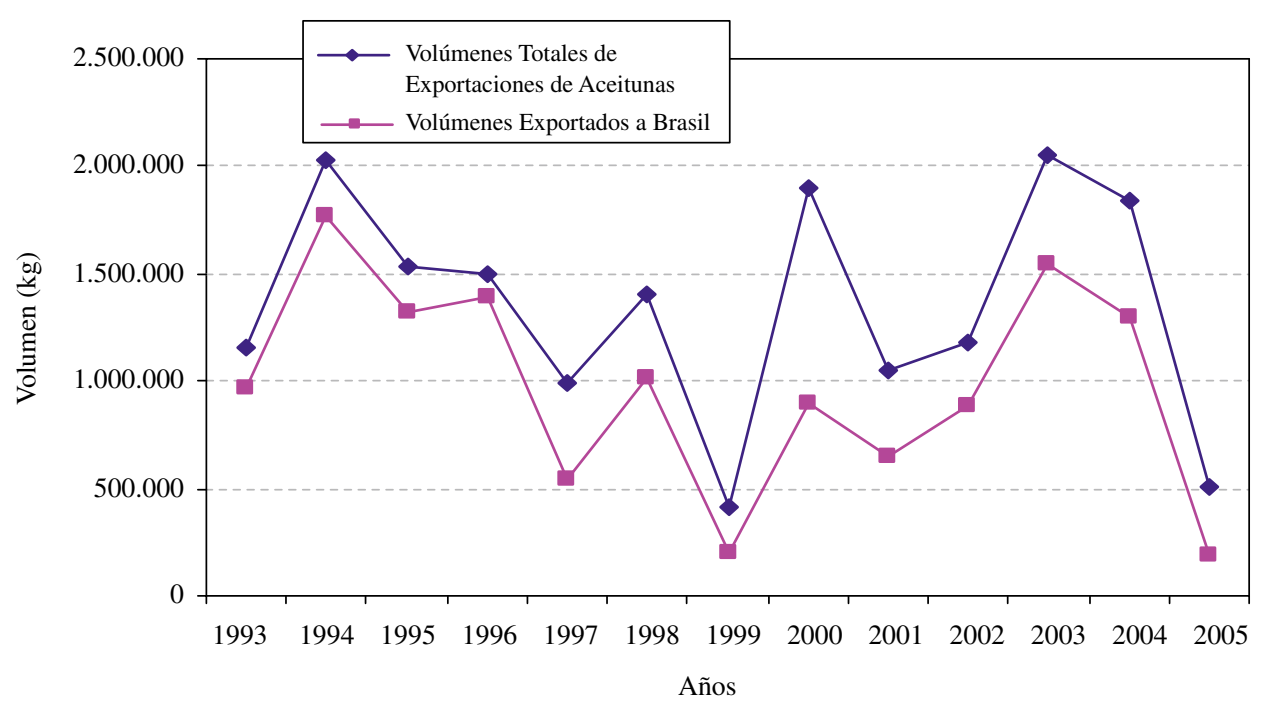

Figura 2. Exportaciones chilenas totales de aceituna de mesa y las exportaciones de este país al mercado brasileño (expresado en $\mathrm{kg})$.

Fuente: Elaboración propia sobre la base de cifras de ODEPA. 1993-2005.

géneas, excepto el año 2000 que, como lo demuestra el gráfico, las importaciones fueron menores.

En la Figura 3 se puede observar la participación de las exportaciones de aceituna de mesa chilena al mercado brasileño en forma porcentual, llegando éstas a su máximo el año 1996, con un 98,2\% de participación y correspondiendo el mínimo al año 2005, con un $37 \%$ de participación. Esta es una ostensible baja en un transcurso de 9 años, con una clara tendencia negativa.

Estas cifras indican una tendencia negativa que se expresa en una pérdida de presencia de aceitunas de mesa en el mercado brasileño. El coeficiente de correlación lineal es 0,58 , lo cual indica que existe una mediana correlación entre las variables volúmenes de exportación de aceituna de mesa chilena al mercado brasileño y el tiempo que se realizan estas exportaciones. Este nivel de correlación es significativo al 5\% Little y Hills (1976).

En la Figura 4 se pueden observar las grandes diferencias de las exportaciones de aceituna de mesa al mercado brasileño registradas entre Chile y Argentina, teniendo este país el año 1994 un máximo de exportación a Brasil de $58.468 \mathrm{t}$, a diferencia de Chile que su máximo fue de $1.621 \mathrm{t}$ en el año 2003.

En consideración a los grandes volúmenes de las exportaciones argentinas al mercado brasileño, se puede consignar que Chile no constituye una competencia directa para Argentina en las exportaciones de aceituna de mesa al mercado brasileño. Asimismo, el 2001 corresponde al año en que las exportaciones argentinas presentaron el menor valor, alcanzando $29.940 \mathrm{t}$, a diferencia de Chile que en 1999, año de menores volúmenes exportados al mercado brasileño, éstas alcanzaron $354 \mathrm{t}$.

En la Figura 5 se puede ver la evolución positiva que han tenido las exportaciones peruanas de aceituna de mesa al mercado brasileño, a diferencia de las exportaciones de aceituna de mesa chilena que en los años 1993, 1994 y 1995 superaban a las exportaciones de aceituna de mesa peruanas orientadas a este mercado.

Se puede observar el gran crecimiento en las exportaciones de aceituna de mesa peruanas, las cuales han variado entre 443,8 t en el año 1993 a $5.918 \mathrm{t}$ en el año 2001. Esta cifra, considerada en el período de tiempo estudiado, es muy superior al máximo de Chile que sólo llegó a 1.621 t en el año 2003.

En la Figura 6 se presenta la evolución de las exportaciones de aceituna de mesa peruanas al mercado brasileño. Esta presenta una tendencia positiva a través de los años, con un mínimo el año 1993 con 443,8 t, y un máximo el año 2005 con 6.406 t, lo cual demuestra la favorable evolución que han tenido 


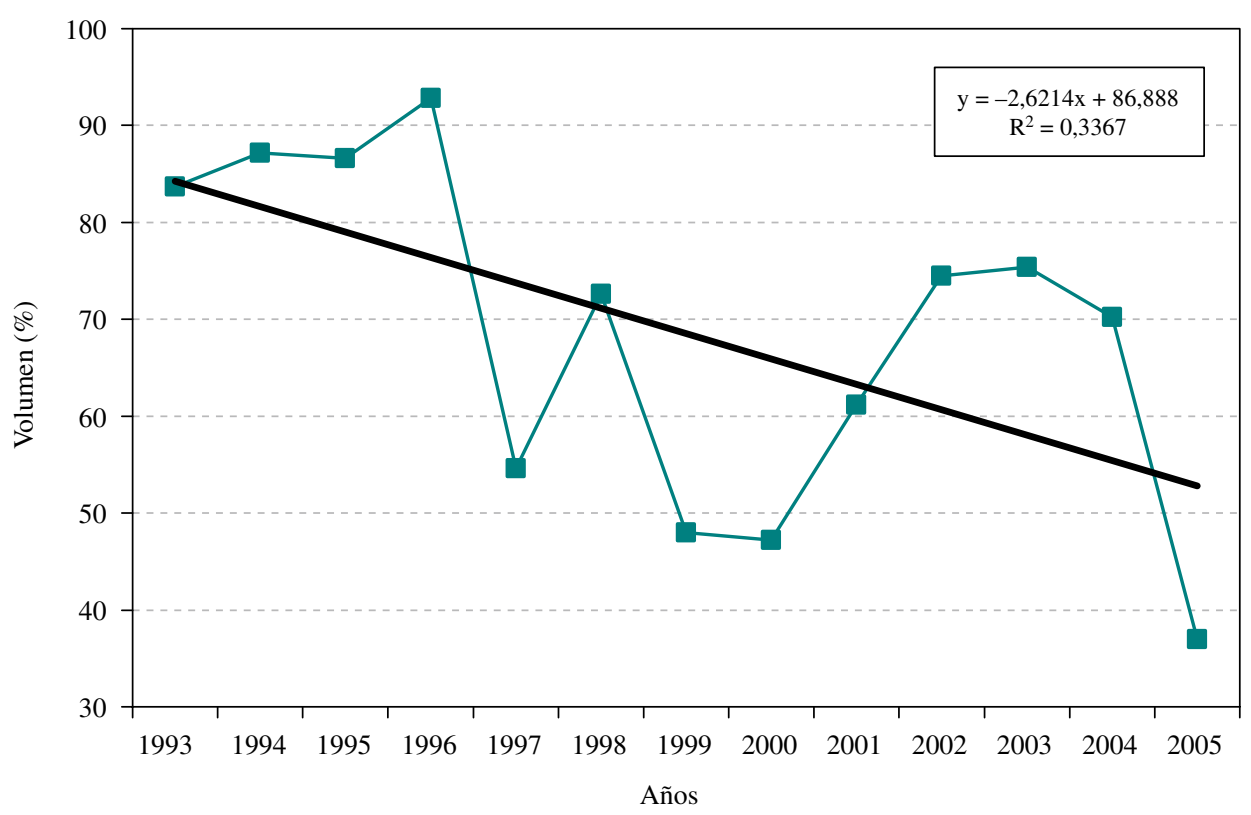

Figura 3. Participación de las exportaciones de aceituna de mesa chilena al mercado brasileño entre los años 1993-2005 (expresado en porcentaje).

Fuente: Elaboración propia sobre la base de cifras ODEPA. 1993-2005.

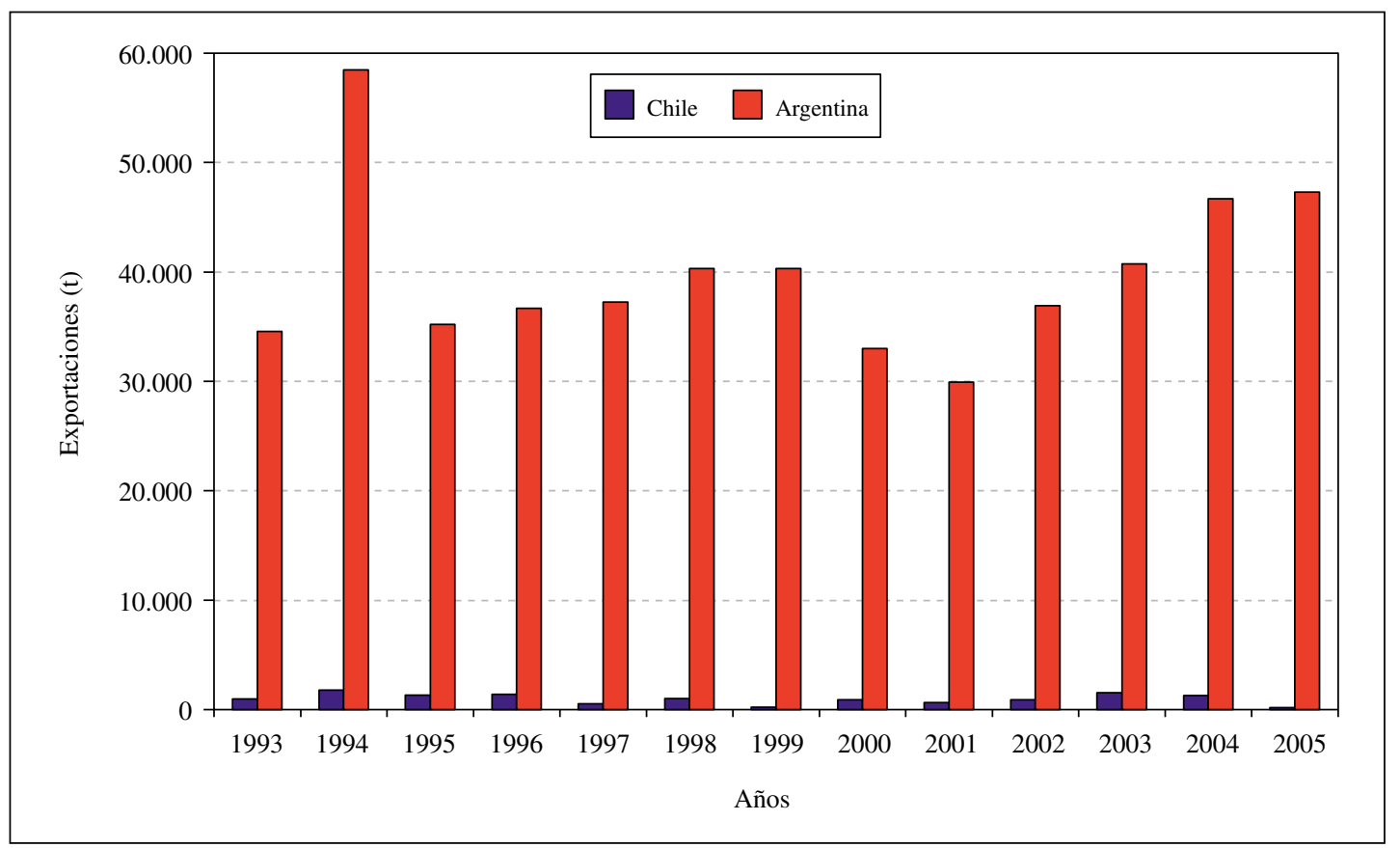

Figura 4. Exportaciones totales de aceituna de mesa por parte de Chile y Argentina al mercado brasileño entre los años 1993-2005 (expresado en toneladas).

Fuente: Elaboración propia sobre la base de cifras de FAO. 1993-2005. 


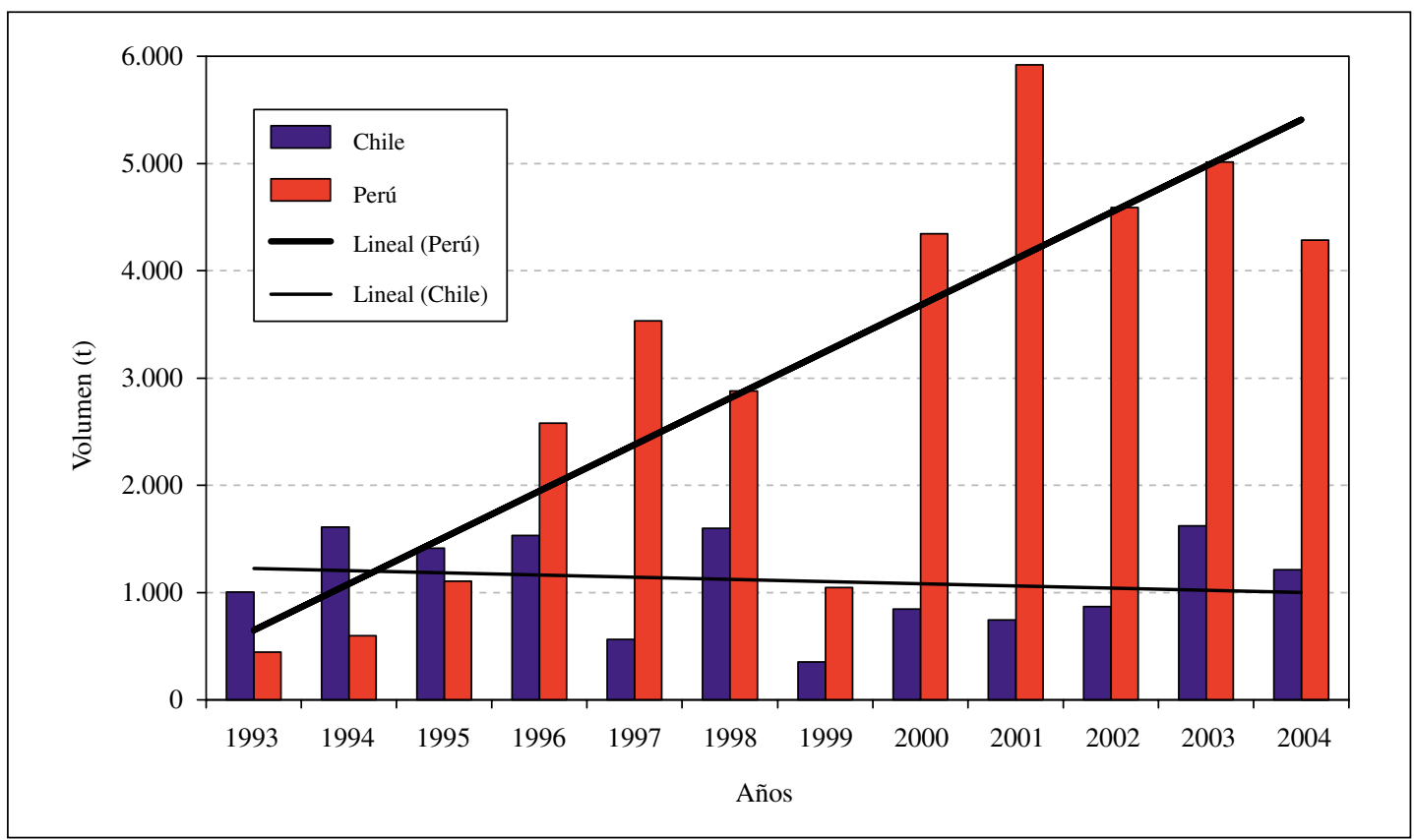

Figura 5. Exportaciones totales de aceituna de mesa al mercado brasileño por parte de Chile y Perú entre los años 1993-2004 (expresado en toneladas).

Fuente: Elaboración propia sobre la base de cifras de FAO. 1993-2004.

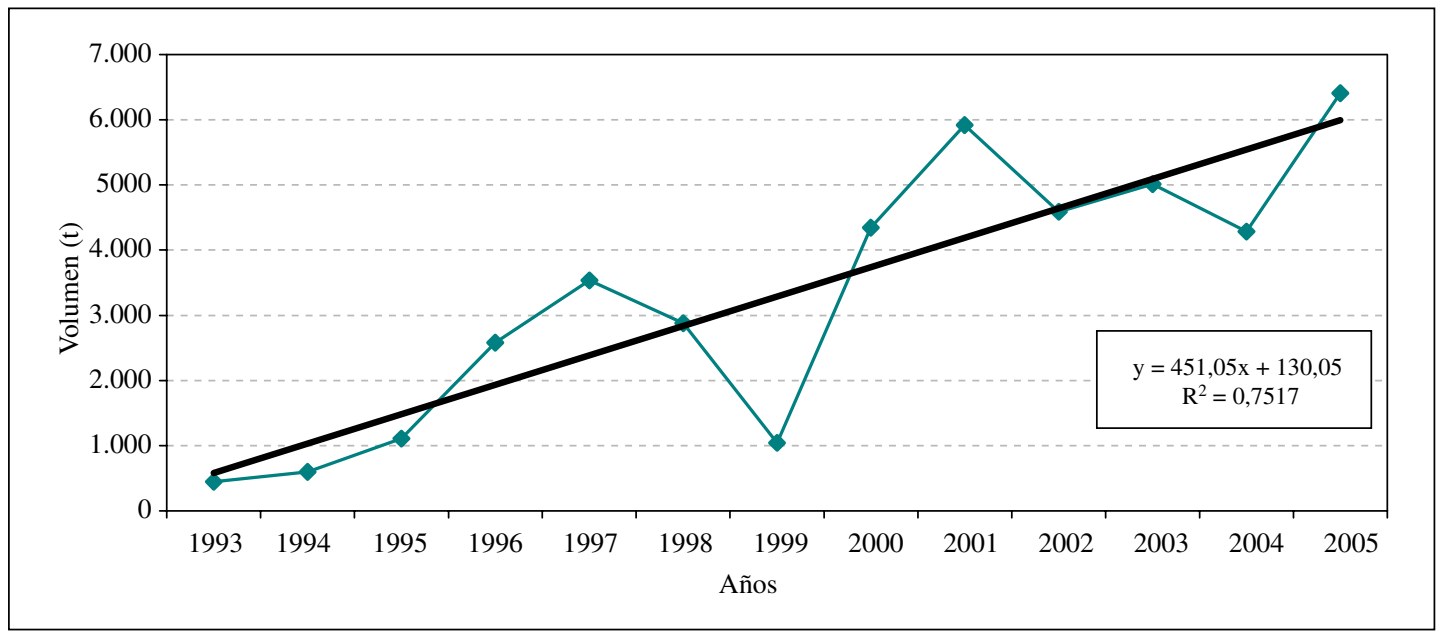

Figura 6. Tendencia de crecimiento de las exportaciones peruanas de aceituna de mesa a Brasil entre los años 1993-2005 (expresado en toneladas).

Fuente: Elaboración propia sobre la base de cifras de FAO. 1993-2005.

las exportaciones olivícolas peruanas al mercado brasileño. Esto puede deberse a la profundización de acuerdos comerciales entre Perú y Brasil, donde gran parte de los productos exportados al mercado brasileño registran preferencias comerciales.

La profundización del Acuerdo de Arancel Parcial - Complementación Económica (AAP.CE) entre Perú y Brasil, expresados en el D.S. N ${ }^{\circ}$ 015-2001 del Ministerio de Industria, Turismo, Integración y Negociaciones Comerciales Internacionales del Perú (ITINCI), ha generado nuevas oportunidades de negocios para los exportadores peruanos y en particular para los olivicultores en el marco de los 
acuerdos de la Asociación Latinoamericana de Integración ALADI (2004).

Los productos agropecuarios no tradicionales peruanos crecieron $52 \%$ en el 2000 , resultado asociado al dinamismo de las exportaciones de aceitunas conservadas provisionalmente y aceitunas preparadas o conservadas sin congelar, las mismas que se adscriben dentro del AAP.CE $\mathrm{N}^{\circ} 39$ con un margen porcentual de desgravamen del 100\%.

A estas condiciones de mercado se agrega que Perú tiene ventajas comerciales como aceitunas de mesa de alta calidad de exportación, disponibilidad de mano de obra barata y rendimientos superiores a la media mundial. Todos estos factores hacen que las aceitunas peruanas se hayan posicionado fuertemente en el mercado brasileño.

El coeficiente de correlación de 0,83 indica una alta correlación entre las variables exportaciones peruanas al mercado brasileño y el tiempo en que se realizaron estas exportaciones. Este valor de correlación es significativo al 1\%, Little y Hills (1976).

En la Tabla 3 se pueden observar los valores de las exportaciones de aceituna a nivel mundial. España es el país que presenta los valores más bajos respecto a los otros exportadores de aceituna de mesa al mercado brasileño, con una media de 0,386 (1.000 US\$ t), seguido por Portugal y Argentina, con una media de 0,315 y 0,688 (1.000 US\$ t), respectivamente.

Tanto Chile como Perú presentan altos valores por toneladas. Perú es el país que presenta los mayores valores de los países exportadores de aceitunas de mesa al mercado brasileño, con una media de 1,159 (1.000 US\$ t), seguido por Chile, con una media de 1,028 (1.000 US\$ t), lo cual no influye en los volúmenes exportados al mercado brasileño, al contrario, se ratifica con la tendencia positiva que tiene Perú en sus exportaciones de aceituna de mesa al mercado brasileño.

\section{CONCLUSIONES}

A continuación se presentan los resultados obtenidos del estudio realizado para conocer la realidad competitiva de la aceituna de mesa chilena frente a sus competidores directos en el mercado brasileño.

Tabla 3

Valor unitario estandarizado de las exportaciones (expresado en 1.000 US\$ por tonelada)

\begin{tabular}{|c|c|c|c|c|c|}
\hline Años & Argentina & Chile & España & Perú & Portugal \\
\hline 1993 & 0,6 & 1,21 & 0,37 & 1,18 & 0,26 \\
\hline 1994 & 0,65 & 1,15 & 0,38 & 1,59 & 0,27 \\
\hline 1995 & 0,84 & 1,61 & 0,53 & 1,56 & 0,3 \\
\hline 1996 & 0,9 & 1,58 & 0,63 & 1,42 & 0,47 \\
\hline 1997 & 0,88 & 1,22 & 0,37 & 1,28 & 0,33 \\
\hline 1998 & 0,76 & 1,07 & 0,32 & 1,35 & 0,28 \\
\hline 1999 & 0,73 & 1,02 & 0,39 & 0,95 & 0,25 \\
\hline 2000 & 0,7 & 0,78 & 0,29 & 0,8 & 0,24 \\
\hline 2001 & 0,65 & 0,67 & 0,26 & 0,75 & 0,24 \\
\hline 2002 & 0,5 & 0,67 & 0,27 & 0,77 & 0,31 \\
\hline 2003 & 0,44 & 0,57 & 0,34 & 0,92 & 0,38 \\
\hline 2004 & 0,68 & 0,85 & 0,4 & 0,97 & 0,4 \\
\hline 2005 & 0,61 & 0,97 & 0,47 & 1,159 & 0,315 \\
\hline Media & 0,688 & 1,028 & 0,386 & & \\
\hline
\end{tabular}

Fuente: Elaboración propia sobre la base de cifras de FAO. 1993-2005. 
- Las exportaciones chilenas de aceituna de mesa tanto al mercado brasileño como al resto del mundo muestran un inestable comportamiento. Brasil es el principal importador de éstas. Una baja en las importaciones de aceituna de mesa por parte de Brasil influye drásticamente en las exportaciones chilenas.

- Argentina es el mayor exportador de aceitunas de mesa al mercado brasileño, siendo su máximo de $58.468 \mathrm{t}$, esta cifra es muy superior a los máximos de exportación alcanzados por Chile, de sólo 1.621 t. Los grandes volúmenes exportados por Argentina al mercado brasileño dominan sin competencia directa este mercado; también hay que considerar que Argentina por ser un país miembro del MERCOSUR, no paga el Arancel Externo Común (AEC) para vender dentro del mercado que genera este grupo económico.

- La evolución de las exportaciones peruanas de aceituna de mesa al mercado brasileño han crecido en los últimos 12 años aproximadamente un $641 \%$, mostrando una tendencia positiva. Esta situación ayuda a explicar la tendencia a la baja que en ese mismo período de tiempo han tenido las exportaciones de aceituna de mesa chilenas a Brasil.

- Además de la competencia de América Latina, está la competencia de países con tradición productora y exportadora olivícola como España o Portugal, que sin exportar grandes volúmenes al mercado brasileño, éstos son estables en el tiempo. Además sus volúmenes son muy similares a los volúmenes exportados por parte de Chile a Brasil.

- En lo que respecta al aspecto de la calidad de la aceituna de mesa que se exporta al mercado brasileño hay que considerar que los productores cumplen con las normas establecidas para un producto de calidad por parte del mercado brasileño y estas exigencias rigen para todos los países competidores.

\section{LITERATURA CITADA}

ASOCIACIÓN LATINOAMERICANA DE INTEGRACIÓN, ALADI. 2004. XIII Reunión del Consejo de Ministros de la Asociación Latinoamericana de Integración. Disponible en: http://www.aladi.org/nsfaladi/aladiorg.nsf/vsitioweb/b oletin15?OpenDocument\&AutoFramed

CONSEJO OLIVÍCOLA INTERNACIONAL, COI. 2005. Revista Olivae, Número 104. Editorial Eumedia. España.

GUERRERO, A. 1997. Nueva Olivicultura. Editorial MundiPrensa. Madrid.

HERNÁNDEZ, R.; FERNÁNDEZ, C.; BAPTISTA, P. 2003. Metodología de la investigación. McGraw-Hill. México.

LITTLE, T.; HILLS, J. 1976. Métodos estadísticos para la investigación en la agricultura. Editorial Trillas. México.
OFICINA DE ESTUDIOS Y POLÍTICAS AGRARIAS, ODEPA. 1993-2005. Base de datos de exportaciones agropecuarias de Chile. Santiago, Chile. Disponible en http://www.odepa.cl

ORGANIZACIÓN DE LAS NACIONES UNIDAS PARA LA AGRICULTURA Y LA ALIMENTACIÓN, FAO. 1993- 2005. Base de Datos Estadísticos, Disponibles en http://fao.stat.org

TAPIA, L.; BASTÍAS, E.; PÉREZ, E. 1985. Incidencia del factor frío invernal en la producción de un huerto olivícola en el valle de Azapa, Primera Región de Chile. IDESIA (Chile) Vol 9. 
